\title{
Leiomyoma of the duodenum presenting as a pyogenic liver abscess
}

\author{
A. Selvam \\ M.B.B.S.
}

L. J. DONALDSON
M.Sc., F.R.C.S. (Edin.)

G. S. SoKHI

F.R.C.S.(Edin.)

\begin{abstract}
Summary
Leiomyoma of the duodenum is a rare condition which is most often diagnosed only as an incidental finding at post-mortem. It may present clinically with pain or haemorrhage. A case is described of degeneration of a leiomyoma of the fourth part of the duodenum giving rise to pyogenic liver abscess. There is no previous report of this association in the literature.
\end{abstract}

\section{Introduction}

Leiomyoma of the upper gastrointestinal tract is a rare condition, most often discovered as an incidental finding at post-mortem with a frequency of $1.17 \%$, the most common site being the stomach $(0.95 \%)$, less commonly the oesophagus $(0.22 \%)$ and only rarely the duodenum (Kavlie and White, 1972). When leiomyoma of the duodenum presents clinically, the symptoms may be pain (Wilson et al., 1975), acute or chronic bleeding (Wilson et al., 1975; Keighley and Pratt, 1976).

In this paper, a case is reported of leiomyoma of the fourth part of the duodenum presenting as a pyogenic liver abscess.

\section{Case report}

A 54-year-old man, who had previously been in good health, was admitted to hospital with pyrexia. Apart from a history of sweating attacks and occasional rigors, he had no other symptoms. On examination, he was pyrexial $\left(39.8^{\circ} \mathrm{C}\right)$, pale and looked very ill. He was not jaundiced but there was a tender smooth enlargement of the liver. Investigations showed: leucocytosis, $16.3 \times 10^{\circ} / 1$; ESR, 108; total bilirubin, $20 \mu \mathrm{mol} / 1$; alkaline phosphatase, 1489 i.u./l; SGOT, 74 i.u./l; SGPT, 84 i.u./l. Blood cultures were negative and chest and abdominal X-rays were unhelpful. A diagnosis of hepatic abscess was made and a laparotomy carried out.

At laparotomy, an intra-hepatic abscess was found involving the right lobe. In addition, there was a mass with surrounding oedema arising from the duodeno-jejunal junction which was adherent to the posterior abdominal wall. A full laparotomy revealed no other possible intra-abdominal source of sepsis. In view of the extremely poor condition of the patient, further exploration of this lesion was not

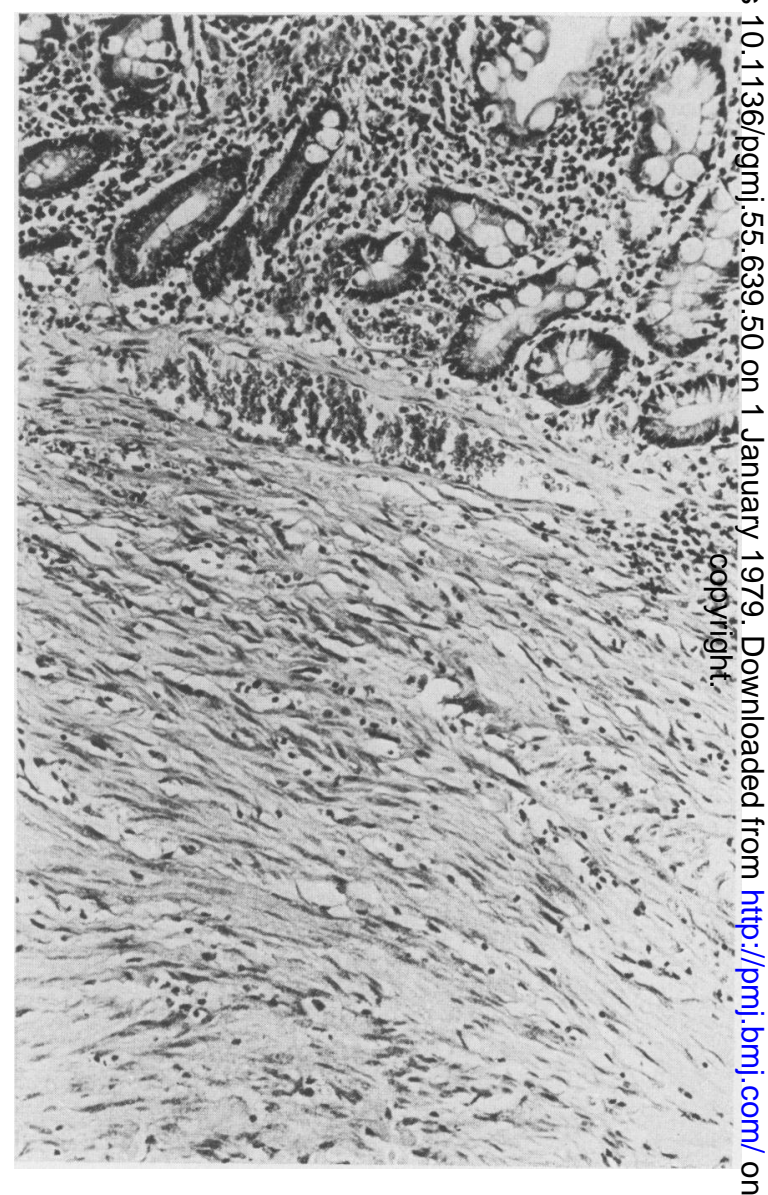

FIG. 1. Photomicrograph of a benign leiomyoma of the fourth part of duodenum. (H \& $\mathrm{E} \times 100$.)

attempted and the liver abscess was drained. Post- $\sigma$ operatively, the patient improved slowly but steadily $N$ and made a full recovery. Four months later, he N underwent further laparotomy to determine the definitive nature of the lesion previously seen. The mass was fully exposed and found to be arising from the fourth part of the duodenum adjacent to the duodeno-jejunal junction. It appeared to be localized with no invasion of surrounding tissues. 
The duodeno-jejunal junction was exposed and the lesion was completely removed with part of the duodenal wall, the defect being closed. The patient made an uneventful post-operative recovery. Pathology of the operative specimen (Fig. 1) showed the histological appearances of a spindle cell tumour of smooth muscle origin, foci of suppuration were present but there was no ulceration of the bowel mucosa. The pathological diagnosis was that of a benign leiomyoma which had undergone degeneration and infection; there was no evidence of malignancy.

\section{Discussion}

In the case described in this paper, a leiomyoma of the fourth part of the duodenum was discovered in a patient undergoing laparotomy and drainage of hepatic abscess. Pyogenic liver abscess is itself an uncommon condition in the United Kingdom, and may be associated with intra-abdominal sepsis such as appendicitis (Ranson et al., 1975) or diverticulitis (Wallack et al., 1976), although Butler and McCarthy (1969) could find no primary site for the sepsis in just under $50 \%$ of the cases studied.

The evidence for a causal relationship between duodenal leiomyoma and hepatic abscess in the case described in this paper is difficult to refute. No other intra-abdominal site of sepsis was found at laparotomy and the oedema and adherence to the sur- rounding mesentery together with its proximity to the portal circulation, would seem to implicate the leiomyoma of the duodenum. Moreover, the histological appearances were those of degeneration with suppuration, and septicaemia has been reported following degenerating leiomyoma of the uterus (Kaufmann, Cooper and Cookson, 1974). To the authors' knowledge this is the first reported case of leiomyoma of the duodenum presenting as pyogenic liver abscess.

\section{References}

Butler, T.J. \& McCarthy, C.F. (1969) Pyogenic liver abscess. Gut, 10, 389.

Kaufmann, B.M., Cooper, J.M. \& Cookson, P. (1974) Clostridium perfringens septicemia complicating degenerating uterine leiomyomas. American Journal of Obstetrics and Gynecology, 118, 877.

Kavlie, H. \& WhITE, T.T. (1972) Leiomyomas of the upper gastrointestinal tract. Surgery, 71, 843.

KeighleY, M.R.B. \& Pratt, D. (1976) Leiomyoma of the duodenum. A cause of recurrent haematemesis. Journal of the Royal College of Surgeons of Edinburgh, 21, 299.

Ranson, J.H.C., MadaYaG, M.A., Localio, S.A. \& SPENDER, F.C. (1975) New diagnostic and therapeutic techniques in the management of pyogenic liver abscesses. Annals of Surgery, 181, 501.

Wallack, M.K., Brown, A.S., Austrian, R. \& Fitts, W.T. (1976) Pyogenic liver abscess secondary to asymptomatic sigmoid diverticulitis. Annals of Surgery, 184, 241.

Wilson, J.M., Melvin, D.B., Grayo, G. \& Thorbuarnarson, B. (1975) Benign small bowel tumor. Annals of Surgery, 181, 247. 